\title{
IOT Based Patient Monitoring in Industrial Areas
}

\author{
K.R.Jansi, Mahesh Srivinay Rayavarapu, Vamsi Koganti
}

\begin{abstract}
With the broadening technology, the usage of several kinds revolutionary advancements in medicine industry are evolving day by day. These advancements have become more accurate than the existing manual procedures and they are reducing the usage of manpower. By using various technologies in the required medical fields, the time taking procedures are becoming handy in lesser time. In this paper, we are going to propose some technological advancements in field of medical sciences, which are mainly useful for the people who are all living and working in industrial areas. Here we are using Sensors and RF communication to monitor the Patient who has the wandering behavior. To monitor the patient health, heartbeat sensor and temperature sensor are used. Also, we place the gas sensor in all the public places to monitor the unwanted gas. If a patient enters the dangerous environments, an alert will be given to the patient in the form of a personalized buzzer, so that the patient will get alerted and he will take some precautions while travelling in those dangerous areas.
\end{abstract}

Index Terms: Gas Sensor, Health-Monitoring, Heartbeat Sensor, MEMS Sensor, Microcontroller, Temperature Sensor, Wireless Communication.

\section{INTRODUCTION}

Healthcare is the basic need for every person. As the nation's grow stronger and bigger, technological growth and industrial growth have been increasing in everyday aspect. Every evolution has some drawbacks. Improper healthcare and pollution are one of the major setbacks in modern world. Industries are packed everywhere in the world. studies conformed that the particles emitted from the industries are one of the major causes for pollution. The people who work in the industries and the people who are living near the industrial areas are severely getting effected. In our proposed system, the patient who is admitted in the hospital will get monitored by sensors. MEM sensor, Heartbeat sensor and the temperature senor are the sensors used in patient monitoring in hospitals. Micro electromechanical system sensor is used to measure the XYZ coordinate values which will tell us when

Revised Manuscript Received on July 05, 2019.

K.R.Jansi, Assistant Professor, Department Of CSE, SRM Institute of Science and Technology (formerly known as SRM University), Kattankulathur, Chennai, Tamil Nadu, India.

Mahesh Srivinay Rayavarapu, Bachelor of Technology, Department of Computer Science and Engineering, SRM Institute of Science and Technology, Kattankulathur, Chennai, Tamil Nadu, India.

Vamsi Koganti, Bachelor of Technology, Department of Computer Science and Engineering, SRM Institute of Science and Technology, Kattankulathur, Chennai, Tamil Nadu, India. the patient was suddenly fallen down, we can use this technology to monitor the patients who are roaming outside the hospital.

Heartbeat sensor, which is placed on the index finger of the patient is a clip type sensor. When there is a sudden deflection in the heartbeat a buzzer typed alarm will start ringing at the nurse/doctor's room. By using this tech, we can monitor the patient effectively with lesser manpower. Similarly, for the temperature sensor, when there is a sudden change in patient's temperature an alert will be given to the nurse/doctor's section in the form of an alarm. When the patient got discharged from the hospital, he will be given a personalized buzzer/alarm. Gas detection sensors will be placed in all public places i.e., near signal towers. Initially, when the patient got admitted in the hospital, all his details were stored in the hospitals database which has the Zigbee wireless transmitter. The Zigbee receiver which is placed at the public places will receive the data from the ZigBee transmitter which is placed at the hospital. When a patient entered the zone which contains harmful gases, an alert will be given to him through the personalized buzzer. The patient will get alerted immediately, so that while he takes some safety measurements like wearing an oxygen mask, while travelling through that dangerous routes. The buzzer will ring continuously in the presence of gasses. The buzzer will stop ringing when he entered the safe places. By using this technology, we can prevent an Asthma patient inhaling excess nitrogen, since excess nitrogen causes suffocation.

\section{RELATED WORKS}

E-Health is one of the greatest evolutions in technological development in medical field. These are electronic based applications that can store, record or transmit information used in healthcare treatment. The main drawback of the E-health applications is it cannot transmit the data without internet. M-Health has extraordinary potential since it adds to the advantages of e-health all the benefits related to the ubiquity of mobile devices Although significant advances have been made, m-health is still in its early stages and is evolving in parallel to another very promising concept: smart cities, which are also founded on ICT and aim to tackle local problems, from local economy and transportation to quality of life and e-governance. Local governments are investing in ICT to equip their cities with technological infrastructures able to support ambient intelligence and foster social responsibility and respect for the environment. In this sense, the opportunities for smart cities are boundless, and companies such as IBM and Intel are taking action to consolidate their leadership in this sector. They have 
identified several important areas in which smart cities will play a key role: public safety, energy and utilities, economic development, education social services, and healthcare, among others. In this paper, unless specifically stated, patient refers to patient with wandering behavior. Currently there is considerable interest for making use of different heterogeneous technologies for healthcare applications. However, there are several challenges for making use of different technologies for healthcare applications. First there is a need to consider the specific requirements for the healthcare application. Then there is a need to consider the challenges with specific technologies used for the healthcare application. In particular, there is a need to ensure that the healthcare related services receive priority during events such as legitimate failures, congestion and attacks. So, we use sensor to monitor the patient health, heartbeat sensor and temperature sensor are used. We can transmit the information through Radio Frequency Modules instead of internet and we also use ZigBee modules which are used for high distance communication.

\section{SYSTEM ANALYSIS}

\section{System Architecture:}

In order to remote monitor the patient health in environment, we create a gas monitoring IOT device and integrated to IOT platform to monitor poisonous gas. In order to evaluate the gases which are present in environment, sensors have been used to analyze the amount of hazardous gas and provide an alert message. The system architecture is shown in the below diagram.

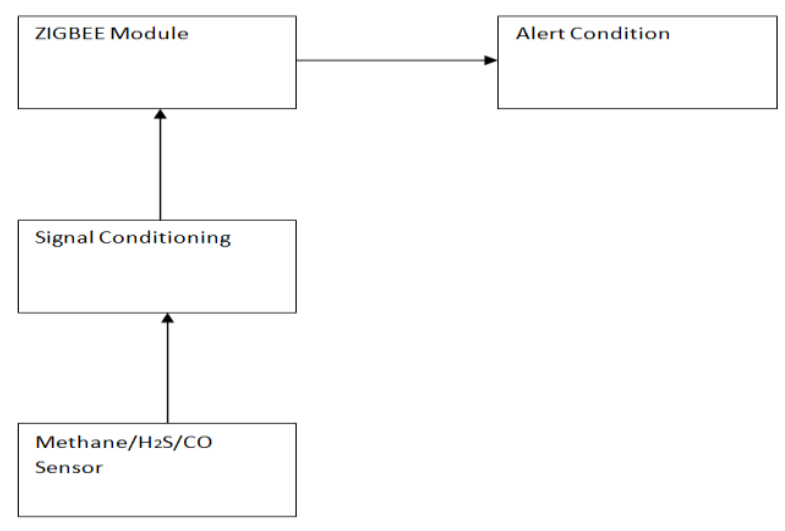

Fig.1: System Architecture

\section{Existing System:}

As per our studies, the patients' health is monitored manually by the doctors and nurses, this is done only in certain time periods as the doctors can't monitor the patient's $24 / 7$. In the existing systems, the patient is not monitored once he is discharged. And there is lack of medicine in Emergency and the medical expansions are more.

The main disadvantages of the existing system is the patient is not monitored $24 / 7$ i.e. no proper patient monitoring. And the time consumption is much more then we expect

\section{Our Approach:}

In the proposed system the patients are monitored who has the wandering behavior. Patient's health is measured by using sensors. The measured information is transferred to doctor using wireless communication by this the patient can be monitored 24/7.Also we place the gas sensor in all the places to monitor the unwanted gas. If any patient enters into the place, the hospital will send the alert message to the patient.

The main advantages of our approach are that patients are monitored properly and continuously. The patients can be monitored even after they are discharged. Patients are monitored through sensors which gives accurate values.

\section{Requirement Specification:}

Various hazardous gases, such as $\mathrm{CO}, \mathrm{CH}_{4}$, and $\mathrm{H}_{2} \mathrm{~S}$ gases are found in worksites and various places that might cause illnesses and fatalities. Table I, lists the advisable threshold levels.

\section{Table.1: Gases and Their Properties}

\begin{tabular}{|c|l|l|l|}
\hline $\begin{array}{l}\text { Hazardous } \\
\text { gas }\end{array}$ & $\begin{array}{l}\text { Lower } \\
\text { threshold } \\
\text { level }\end{array}$ & $\begin{array}{l}\text { Upper } \\
\text { threshold } \\
\text { level }\end{array}$ & $\begin{array}{l}\text { Odor } \\
\text { and } \\
\text { Color }\end{array}$ \\
\hline $\mathrm{H}_{2} \mathrm{~S}$ & 2300 & 3500 & $\begin{array}{l}\text { Rotten } \\
\text { egg }\end{array}$ \\
\hline $\mathrm{CO}$ & 4500 & 7500 & $\begin{array}{l}\text { Odorless } \\
\text { and } \\
\text { Colorles } \\
\text { s }\end{array}$ \\
\hline $\mathrm{CH}_{4}$ & & 2300 & $\begin{array}{l}\text { Displace } \\
\text { air } \\
\text { causing } \\
\text { asphyxia } \\
\text { tion }\end{array}$ \\
\hline
\end{tabular}

The threshold limit of various harmful gas sensors like carbon monoxide, Hydrogen sulfide and Methane can be varied depends on level of gas occurred. If the gases are beyond threshold limit, workers or patients may lead to death. Therefore, the threshold limit is measured based on gas levels low, medium and high the threshold limit is measured. The maximum gas value such as high level is said to be a threshold limit.

\section{Signaling Condition:}

In order to convert the sensed gases using various sensors, each sensor is configured with amplifying circuit using opamp and a potentiometer is used to calibrate the sensed signals from each sensor such as Methane/H2S/CO.

\section{RF Module:}

RF module mainly divided into two subdivisions, a transmitter and a receiver. Radio frequency transmitter is a small electronic device which will transmit and receive information in the form of radio signals.

Some radio frequency modules can transmit up to 
$500 \mathrm{ft}$. Not only radio frequency communication, these modules use optical communication also. It can be of any shape and size.

Transmitter module will transmit a radio wave and modulate that wave to carry data. Whereas, the receiver module receives the modulated rf signal transmitted from the transmitter and demodulates it. RF Transceiver is also one of the radio frequency modules which can act both transmitter and receiver.

\section{Gas Sensor:}

A gas sensor is a device which detects the presence of gasses in an area. These sensors have a high sensitivity for detecting gas concentrations i.e. which makes them widely used for leak detection. In our case the gas sensors are placed in public places to detect the poisonous gases and send an alert message to the patient when entered the area.

\section{MEMS Sensor:}

MEMS, Micro-Electro-Mechanical systems, is the ultimate enabling technology for the integration of almost any physical, chemical and biological phenomena that include motion, light, sound, chemistry, biochemistry, radio waves, and computation, but all on a single chip. The MEMS sensors are tiny chips that are used for mechanical sensing, control and motion to solid state electronics to deliver extraordinary functionality. These sensors can sense pressure, detect motion and various other functions in medical fields.

In our project, we are using MEMS sensor to detect motion of the patient by using co-ordinates. We will be attaching the MEMS sensor to the patient, by this we can monitor him. If the patient drops suddenly the sensor will detect the motion of the patient and alert the hospital management. By this, the hospital management can attend the patient as fast as possible.

\section{PIC Development Board:}

Here the PIC stands for Peripheral interface controller. In our proposed project we are using two PIC development boards, one for hospital section and other for public places section. These are main parts of the system, without the microcontroller the data can't be recorded, transmitted or received. We connect all the sensors i.e. MEMS sensor, Heartbeat sensor, Temperature sensor and Gas sensors to the microcontroller through wires.

The main advantage of PIC microcontroller is it consumes low power. In PIC microcontroller the flash technology is used which allows the data to retain even after the power is switched off.

\section{Block Diagram:}

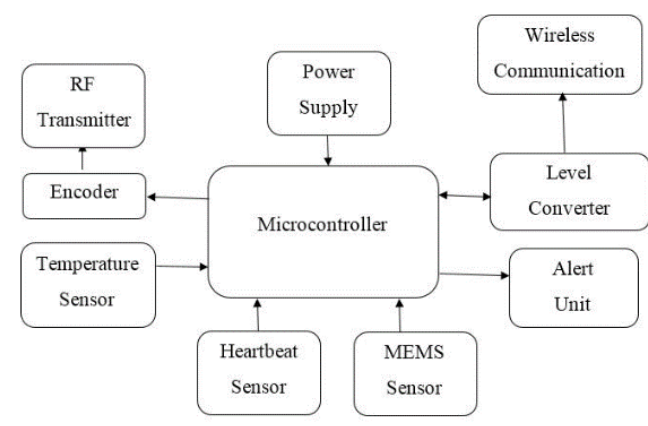

Fig.2: Patient section

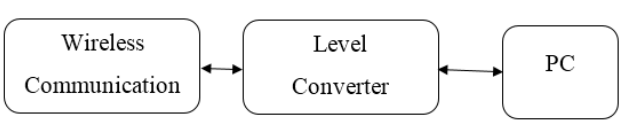

Fig.3: Hospital Section

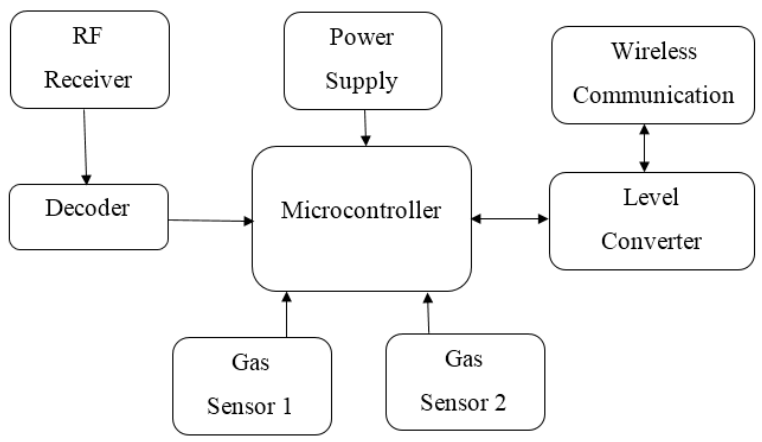

Fig.4: Public Places Section

\section{Patient section:}

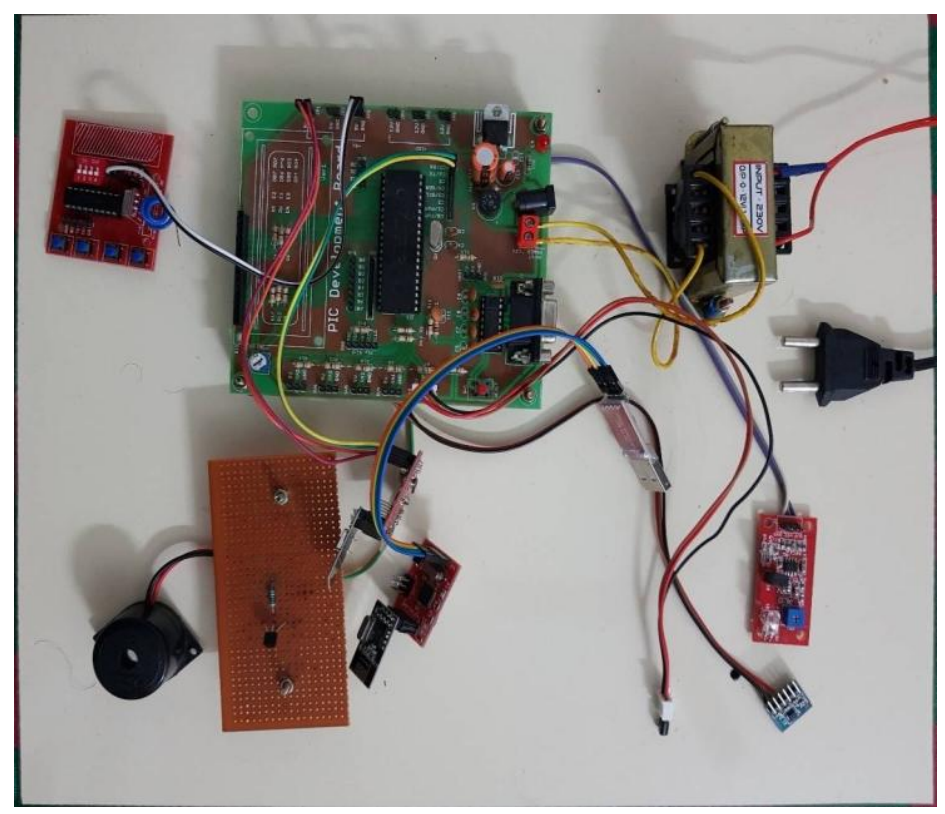

Fig.5: Patient Section 
In the patient section, we use sensors, RF transmitter and wireless communication.

The RF transmitter transmits the patient information to the RF receiver. The RF transmitter transmits the encoded data, so we use the encoder with the RF transmitter.

Here we use the sensors like temperature sensor, Heartbeat sensor and the MEMS sensor to measure the person's health. The measured information is transferred to the Hospital by using the Wireless communication. Here we are using Zigbee for the wireless communication.

\section{Hospital Section:}

The Hospital section contains the Wireless communication with the PC. For the wireless communication we use the Zigbee Module. The Zigbee is the transceiver. So we can do both transmit and receive the data.

The transmitted information is received by the hospital section and the information is showed by the PC.

\section{Public Place:}

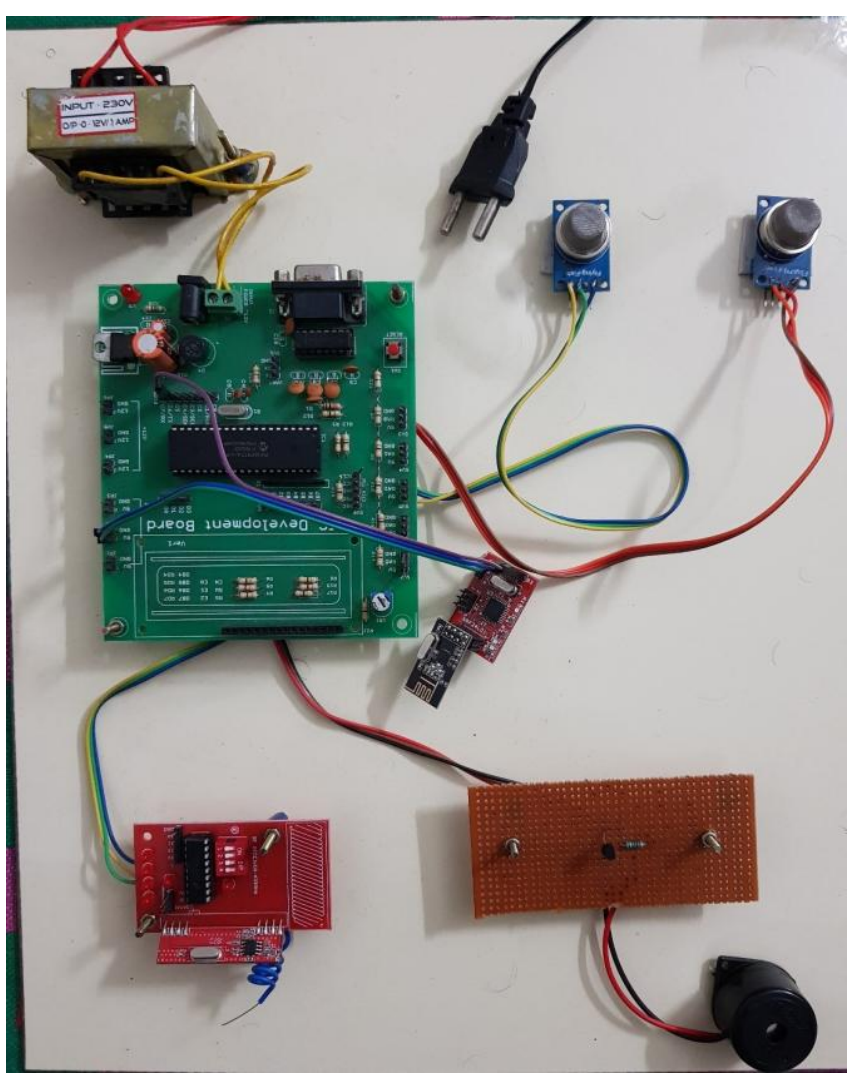

Fig.6: Public Places Section

This module contains the RF receiver, gas sensor and the wireless communication.

The gas sensor is used to sense the unwanted gas in other places.

A radio frequency receiver is placed at public places i.e. traffic signal, buildings, towers etc. The radio frequency transmitter which is placed at the hospital transmits the data to the receiver where the encoded data will be decoded. If the person is the patient, then the wireless communication sends the information to the hospital. And also, the alert message will get $\mathrm{ON}$ to alert the patient.

\section{CONCLUSION}

In this paper we have proposed a technique to lower the usage of manpower by increasing technical assistance in medical field. By this we can monitor the patient in real time, securely and we can prevent the further damage. By using our proposed technique, it lowers the cost involved and increases efficient usage of data. The wandering patients can also be monitored through sensors inside hospital and even after discharging. As a result of this patients can be monitored accurately and can experience highest quality medical care in their own homes and in public.

\section{REFERENCES}

[1] C. Liu and J. Yu, "A solution to wlan authentication and association dos attacks," IAENG International Journal of Computer Science, vol. 34, no. 1, pp. 31-36, 2007.

[2] P. Dawson and D. W. Reid, "Behavioral dimensions of patients at risk of wandering," The Gerontologist, vol. 27, no. 1, pp. 104-107, 1987.

[3] C. K. Lai and D. G. Arthur, "Wandering behavior in people with dementia," Journal of advanced nursing, vol. 44, no. 2, pp. 173-182, 2003.

[4] D. L. Algase, E. R. Beattie, and B. Therrien, "Impact of cognitive impairment on wandering behavior," Western Journal of Nursing Research, vol. 23, no. 3, pp. 283-295, 2001.

[5] C. Iadecola, "The pathobiology of vascular dementia," Neuron, vol. 80, no. 4, pp. 844-866, 2013.

[6] B. Jordan. (2013) Emergency services find lost dementia suffere.http://www.greatfete.com.au/media/NewsLocal-Hills ShireTimes-13Aug2013-Page3.pdf.

[7] S. M. R. Islam, D. Kwak, M. H. Kabir, M. Hossain, and K. S. Kwak, "The internet of things for health care: A comprehensive survey," IEEE Access, vol. 3, pp. 678-708, 2015.

[8] M. Alhussein, "Monitoring parkinson x2019; s disease in smart cities," IEEE Access, vol. PP, no. 99, pp. 1-1, 2017.

[9] M. Chen, Y. Hao, K. Hwang, L. Wang, and L. Wang, "Disease prediction by machine learning over big data from healthcare communities," IEEE Access, vol. 5, pp. 8869-8879, 2017.

[10] O. N. Foundation, "Software-defined networking: The new norm for networks," https://www.opennetworking.org/ images/stories/downloads/sdnresources/white-papers/ wp-sdn-newnorm.pdf [Accessed12Dec.2015].

[11] A. Shalimov, D. Zuikov, D. Zimarina, V. Pashkov, and R. Smeliansky, "Advanced study of sdn/open flow controllers," in Proceedings of the 9th central \& eastern European software engineering conference in Russia. ACM, 2013, p. 1.

[12] P. Porras, S. Shin, V. Yegneswaran, M. Fong, M. Tyson, and G. Gu, "A security enforcement kernel for open flow networks," in Proceedings of the first workshop on Hot topics in software defined networks. ACM, 2012, pp. 121-126.

[13] H. Li, P. Li, S. Guo, and S. Yu, "Byzantine-resilient secure software defined networks with multiple controllers," in 2014 IEEE International

Conference

Communications (ICC). IEEE, 2014, pp. 695-700. 
[14] S. Shin, Y. Song, T. Lee, S. Lee, J. Chung, P. Porras, V. Yegneswaran, J. Noh, and B. B. Kang, "Rosemary: A robust, secure, and high-performance network operating system," in Proceedings of the 2014 ACM SIGSAC conference on computer and communications security. ACM, 2014, pp. 78-89.

\section{AUTHORS PROFILE}

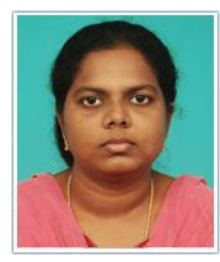

K.R.Jansi, Assistant Professor, Department Of CSE, SRM Institute of Science and Technology (formerly known as SRM University), Kattankulathur, Chennai, Tamil Nadu, India,2005-tilldate. Contact her at: jansik@ srmist.edu.in

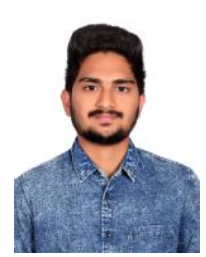

Mahesh Srivinay Rayavarapu, Bachelor of Technology, Department of Computer Science and Engineering, SRM Institute of Science and Technology, Kattankulathur, Chennai, Tamil Nadu, India.

Contact him at: rmsvinay123@gmail.com

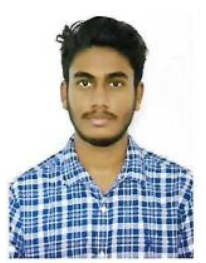

Vamsi Koganti, Bachelor of Technology, Department of Computer Science and Engineering, SRM Institute of Science and Technology, Kattankulathur, Chennai, Tamil Nadu, India. Contact him at: vamsikoganti1@gmail.com 